\title{
Dispersion Equation Obtained by a Semiclassical Model in a Discrete Ferromagnetic System
}

\author{
Breno Rodrigues Segatto \\ Departamento de Ciências Naturais, Universidade Federal do Espírito Santo, São Mateus, ES, Brazil \\ breno.segatto@ufes.br
}

\begin{abstract}
Motived to understanding the behavior of the spin wave propagation in magnetic material, we propose a semiclassical approach to obtain the dispersion equation in a system with $N$ spins in a lattice with parameter $a$ interacting via discrete interaction. We found an unexpected term in the dispersion equation not reported before.
\end{abstract}

\section{Introduction}

In ferromagnetic systems, neighboring spins are coupled by the exchange interaction. Thus, the excitations of the system correspond to collective spin precession around the equilibrium position. The excitation of minor energy is the uniform mode in which spins precession around applied magnetic field $\mathrm{H}$ remaining parallel to each other, i.e. having the same phase [1]. In this case the interaction between the spins do not contribute to the precession frequency. Due to the interaction between the spins, the system also has collective modes in which the phase of precession varies in space, called spin waves. The uniform mode is actually a spin wave with wave length infinite. As the wavelength decreases, the angle between neighboring spins and therefore increases the contribution to the energy exchange excitation energy. The spin waves, among them uniformly, are the el- ementary excitations of a magnetic system. They are quantized, with its quantum called magnon [2]. The magnons are thermally excited and obey BoseEinstein statistics, since the temperature is not close to the critical temperature at which the system passes to the ferromagnetic phase. The spin waves can be excited and detected by a variety of experimental techniques such as magnetic resonance, light scattering and neutron scattering [3, 4, 5, 6, 7, 8]. In our model, we propose a Spin-Spin interaction via a discrete exchange energy in a magnetic field $\mathrm{H}$, where $\mathrm{H}$ includes the external fields plus any molecular field.

\section{Semiclassical Model}

There are some theoretical approach in the literature about spins waves since the 1930s, [2, 9] and the semiclassical and the quantum pictures of spin wave was thoroughly explored [10, 11, 12, 13, 14, 15 , 
16, 17, 18, 19].

We will consider a set of $N$ spins interacting in a solid with lattice parameter $a$ via exchange energy (Heisenberg Hamiltonian)

$$
H=-2 J \sum_{i \neq j} \vec{S}_{i} \cdot \vec{S}_{j}
$$

Where the summation is taken over all nearest neighbors and $J$ is the exchange integral between nearest neighbors. Here we will consider $\vec{S}$ the spin vector of the system with magnitude $S$.

Considering that the system is perturbed from the equilibrium position and the Spin in the site $N$ depends of the configuration of the spin at site $N-1$, and the temporal evolution will be taken account as snapshot pictures at the sites, thus the perturbation in the system at site one is $\vec{S}_{1}=\vec{S}_{0}+\Delta \vec{S}_{0}$ and consequently at site $N$ $\vec{S}_{N}=\vec{S}_{N-1}+\Delta \vec{S}_{N-1}$. Here, we will consider, that the change in the spin vector (the term operator is omitted due the classical approach) $\Delta \vec{S}_{N}$ is proportional to the lattice parameter $a$ and to the momentum transfer $\vec{L}_{N}$. We will postulate that the changes in the momentum transfer are:

$$
\Delta \vec{L}_{N}=-b k \vec{S}(k)=\vec{L}_{N}-\vec{L}_{N-1}
$$

Where $b$ is a constant of proportionality that we will define to be equal one, $k$ is the wave vector, and define the direction of propagation of the perturbation, and $\vec{S}(k) \propto k\left|\vec{S}_{N}\right|$, its consistent since magnons have a parabolic dispersion relation. From this point on we will adopt the notation $|\vec{S}|=S$ and setting

$$
S_{1}=S_{0}+a L_{0}
$$

And the Nth site, the propagation will be:

$$
\begin{aligned}
& S_{N}=S_{N-1}+a L_{N-1} \\
& S_{N}=S_{0}+a \sum_{j=0}^{N-1} L_{j}
\end{aligned}
$$

and consequently

$$
\begin{aligned}
& L_{N}=L_{N-1}-a k^{2} S_{N-1} \\
& L_{N}=L_{0}-a k^{2} \sum_{j=0}^{N-1} S_{j}
\end{aligned}
$$

The recursive combination of equation (5) leads to

$$
S_{N}=\sum_{s=0}^{\left[\frac{N}{2}\right]}(a k)^{2} S_{N}^{(s)}
$$

where $S_{N}^{(s)}$ is a polynomial functions of $S_{0}$, $\left[\frac{N}{2}\right]$ is the largest integer in $\frac{N}{2}$, with

$$
S_{N}^{(0)}=S_{0}+L_{0} \sum_{j_{1}=0}^{N-1} a=S_{0}+L_{0} N a
$$

and

$$
S_{N}^{(s)}=-\sum_{j_{1}=0}^{N-1} \sum_{j_{2}=0}^{j_{1}-1} S_{j_{2}}^{(s-1)}
$$

Successive re-iterations on equation (10) lead to

$$
S_{N}^{(s)}=(-1)^{s} \sum_{j_{1}=0}^{N-1} \sum_{j_{2}=0}^{j_{1}-1} \ldots \sum_{j_{2 s}=0}^{j_{2 s-1}-1} S_{j_{2 s-1}}^{(0)}
$$

$$
S_{N}^{(s)}=(-1)^{S} \sum_{j_{1}=0}^{N-1} \sum_{j_{2}=0}^{j_{1}-1} \ldots
$$

$\sum_{j_{2 s}=0}^{j_{2 s-1}-1}\left(S_{0}+a L_{0} j_{2 s}\right)$

Now, we use $N=\left(\begin{array}{c}N \\ 1\end{array}\right)$ and the identity

$$
\sum_{j=0}^{N-1}\left(\begin{array}{l}
j \\
w
\end{array}\right)=\left(\begin{array}{c}
N \\
w+1
\end{array}\right)
$$


for $N>w$.

This can easily be proved by induction, to get

$$
S_{N}^{(s)}=(-1)^{s}\left(S_{0}\left(\begin{array}{l}
N \\
2 s
\end{array}\right)+a L_{0}\left(\begin{array}{c}
N \\
2 s+1
\end{array}\right)\right)
$$

Which, with equation $(8)$ leads to

$$
\begin{aligned}
S_{N}= & \sum_{s=0}^{\left[\frac{N}{2}\right]}(-1)^{s}\left[(a k)^{2 s} S_{0}\left(\begin{array}{l}
N \\
2 s
\end{array}\right)+\right. \\
& \frac{(a k)^{2 s+1} L_{0}}{k}\left(\begin{array}{c}
N \\
2 s+1
\end{array}\right)
\end{aligned}
$$

For $N \gg 1$, however, the following approximation is valid

$$
\left(\begin{array}{l}
N \\
w
\end{array}\right) \approx \frac{N^{w}}{w !}
$$

And then

$$
\begin{aligned}
& \sum_{s=0}^{\left[\frac{N}{2}\right]}(-1)^{s}(a k)^{2 s}\left(\begin{array}{c}
N \\
2 s
\end{array}\right) \approx \\
& \sum_{s=0}^{\left[\frac{N}{2}\right]}(-1)^{s}(a k)^{2 s} \frac{N^{2 s}}{(2 s) !} \approx \\
& \approx \cos (N a k) \\
& \sum_{s=0}^{\left[\frac{N}{2}\right]}(-1)^{s}(a k)^{2 s+1}\left(\begin{array}{c}
N \\
2 s+1
\end{array}\right) \approx \\
& \sum_{s=0}^{\left[\frac{N}{2}\right]}(-1)^{s}(a k)^{2 s+1} \frac{N^{2 s+1}}{(2 s+1) !} \approx \\
& \approx \sin (N a k)
\end{aligned}
$$

Thus

$$
S_{N}=S_{0} \cos (N k a)+L_{0} \frac{\sin (N k a)}{k}
$$

We can interpret the equation 20 as adjacent spins in a solid with lattice constant $a$ that participate in a mode with wavevector $k$ having an angle between them equal to $k a$.

The model requires a periodic boundary conditions that is equivalent to bending the a chain around into a ring so that first spin is a the $N+1$ spin, each spin $j$ has two nearest neighbors, $j+1$ and $j-1$, with which it interacts trough the exchange integral $J$. We will replaced the quantum pictures of the ladder operators by the wave evolution in $+k$ and $-k$. Consequently we have:

$$
\begin{aligned}
& E_{j}^{(+k)}=-2 J S^{2} \cos (j k a)+ \\
& 2 L_{0} \frac{\sin (j k a)}{k} \\
& E_{j}^{(-k)}=-2 J S^{2} \cos (j k a)+ \\
& 2 L_{0} \frac{\sin (j k a)}{k}
\end{aligned}
$$

The deviations from the ground state $E_{0}$ between adjacent spins, where

$$
E_{0}=-N J S^{2}-g \mu_{B} h S,
$$

will be

$$
\begin{aligned}
& \Delta E=\left(-2 J S^{2} \cos (k a)+2 L_{0} \frac{\sin (k a)}{k}-\right. \\
& \left.g \mu_{B} h S\right)-\left(-4 J S^{2}-g \mu_{B} h S\right) .
\end{aligned}
$$

Consequently the obtained dispersion equation is

$$
\hbar \omega=4 J S^{2}(1-\cos (k a))+4 L_{0} \frac{\sin (k a)}{k} .
$$

This equation show us that the discretization increase the energy of the system that return to the normal behavior after first maximum due to damping naturally present in the last term of the equation . 


\section{Conclusions}

In summary, we propose a discrete semiclassical model, and we obtain the dispersion energy equation of magnons in a system interacting via exchange energy with a large number of sites that can be find requiring a periodic boundary conditions. An analytical solution was proposed that could be helpful in other periodic systems. In a previous paper [20] we were applied this mathematical approach to understanding quantum-to-classical transition and was possible to see in a clear way that this discrete to-continuous transition occurs. We intend in future work to perform some computer simulations to better understand the relationship between a discrete interaction with spin wave propagations.

\section{Acknowledgments}

The author would like thanks Professor Manoelito Martins de Souza for helpful discussions.

\section{References}

[1] D.D. Stancil, A. Prabhakar, Spin Waves, DOI 10.1007/978-0-38777865-52 33 Springer Science + Business Media, LLC 2009.

[2] G.Heller and H.A. Kramers, Proc. R. Acad. Sci. Amsterdam 37, 378 (1934) .

[3] W. Marshall and S. W. Lovesey, Thermal Neutron Scattering, Claredon, Oxford, 1971.

[4] L. J. de Jongh and A. R. Miedema, Experiments on Simple Magnetic
Model Systems,(Taylor \& Francis, London, 1974).

[5] R. M. White, Quantum Theory of Magnetism (Springer-Verlag,Berlin, 1983).

[6] P. A. Fleury, S. P. S. Porto, L. E. Cheesman, and H. J. Guggenheim, Phys. Rev. Lett. 17, 84 (1966).

[7] J. R. Sandercock and W. Wettling, J. Appl. Phys. 50, 7784 (1979).

[8] M. G. Cottam and D. J. Lockwood, Light Scattering in Solids (Wiley, New York, 1986).

[9] F. Bloch, Z. Phys. 61, 206 (1930).

[10] F. Keffer, Handbuch der Physik (Springer Verlag, Berlin, 1966), Vol. $18 / 2$.

[11] B. Lax and K. J. Button, Microwave Ferrites and Ferrimagnetics (McGraw-Hill, New York, 1962).

[12] J. R. Eshbach, J. Appl. Phys. 34, 1298 (1963)

[13] C. Herring and C. Kittel, Phys. Rev. 81, 869 (1951).

[14] A. I. Akhiezer, V. G. Baryakhtar, and M. I. Kaganov, Usp. Fiz. Nauk 71, 533 (1960).

[15] L. R. Walker, in Spin Waves and Other Magnetic Modes, edited by G. T. Rado and H. Suhl, Magnetism Vol. I (Academic Press, New York, 1963)

[16] S. M. Rezende, F. M. de Aguiar, and A. Azevedo, PHYSICAL REVIEW B 73, 094402 (2006).

[17] A. I. Akhiezer, V. G. Baryakhtar, and S. V. Peletminskii, Spin Waves (North-Holland, Amsterdam, 1968). 
[18] C. Kittel, Quantum Theory of Solids (Wiley, New York, 1963).

[19] A. A. Maradudin and D. L. Mills, J. Phys. Chem. Solids 28, 1855
(1967).

[20] Segatto B. R. , Azevedo J. C. S. and de Souza M. M. J. Phys. A: Math. Gen. 36 (2003) $5115 ? 5120$. 